\title{
Effective visco-elastic models of tough, doubly cross-linked, single-network polyvinyl alcohol (PVA) hydrogels
}

\author{
Additively separable fractional derivative-based models for chemical and physical \\ cross-links
}

Received: 4 November 2019 / Accepted: 24 February 2020 / Published online: 7 March 2020

(C) The Author(s) 2020

\begin{abstract}
An effective fractional derivative-based visco-elastic model of tough, doubly cross-linked, singlenetwork polyvinyl alcohol (PVA) hydrogels, embodying both chemical and physical cross-links, is developed using a Mittag-Leffler relaxation function of order $1 / 2$ while applying only three material parameters that are physically quantifiable, namely frequency for maximum loss modulus, equilibrium elastic modulus and relaxation intensity. The resulting 3-parameter shear modulus model is possible to additively split into chemical and physical parts; the split being the study focus. Physical explanations of the visco-elastic low-, mid- and high-frequency range properties, and their transitions between the frequency ranges, are given mainly in terms of the permanent chemical cross-links and the distinct adhesion-deadhesion processes of the transient physical cross-links. The latter are running from an associated Rouse mode low-frequency behaviour through a maximum adhesion-deadhesion dissipation and to an elastic, fully active cross-link high-frequency behaviour, while the former are displaying essentially an elastic, fully active cross-link behaviour throughout the frequency range. The developed model covers the full frequency range while matching measurements results remarkably well. Furthermore, the model is refined into a 4-parameter model by additively including an Abel relaxation function of order $1 / 2$ to take into account the superimposed Rouse-type behaviour found in the measurements of the chemical cross-links in addition to their dominating elastic response, with the fourth parameter being a chemical Rouse stress intensity factor. The simple, effective visco-elastic models are suitable in predicting the mechanical properties of tough, doubly cross-linked, single-network PVA hydrogels with application potentials in tissue and noise abatement engineering.
\end{abstract}

Keywords Doubly cross-linked, single-network hydrogel · Chemical cross-link · Physical cross-link . Mittag-Leffler relaxation function $\cdot$ Rouse mode $\cdot$ Abel relaxation function

\section{Introduction}

Hydrogels are an interesting material, essentially consisting of cross-linked macromolecules containing hydrophilic functional groups while forming a three-dimensional polymer network, being abundantly swollen with water and intrinsically owing application potentials in such as hygienic products, food additives, bio sensors and in tissue engineering $[1,12,14,18,53,69,77,80,82]$ and, more recently, as dampers and vibration isolators [72]. Tissue engineering includes fabrication of artificial cartilages, blood vessels, tendons, bladders, muscles and ligaments. Early fabricated hydrogels are displaying a poor mechanical strength while containing a single polymer network chemically cross-linked by covalent bonds. However, more recent hydrogels are

Communicated by Johlitz, Laiarinandrasana and Marco.

L. Kari $(\varangle)$

The Marcus Wallenberg Laboratory for Sound and Vibration Research (MWL),

Department of Engineering Mechanics, KTH Royal Institute of Technology, 10044 Stockholm, Sweden

E-mail: leifkari@kth.se 
containing a double network; the first network is tightly cross-linked, while the second is loosely cross-linked, as successfully developed by Gong et al. $[18,19]$ and other $[2,23,76]$. Those recent hydrogels are displaying a substantial increase in mechanical strength needed for the previously mentioned artificial body parts.

However, double-network hydrogels with only chemical bonds display permanent strain softening and loss of mechanical strength at repeated loading and unloading due to permanent rupture of chemical crosslinks, mainly located at the tightly cross-linked network. The permanent mechanical strength is substantially increased for double-network hydrogels where chemical cross-links, located at the tightly cross-linked network, are substituted by physical cross-links $[6-8,14,25,28,38,47,52,53,63,73,75,77,82]$. In principle, the physical cross-links broken dissipate energy and act as sacrificing cross-links at loading while being reassembled at unloading, thus leading to recovery, where the second, chemically cross-linked network provides a memory of the initial undeformed state and prevents propagation of cracks $[6-8,14,25,28,38,47,52,53,63,73,75,77,82]$. Physical cross-links may include hydrogen bonds, hydrophobic, $\pi-\pi$, van der Waals and ionic; ion-ion, ion-dipole, dipole-dipole interactions, see e.g. Refs. $[1,45,69,80,82]$.

Large deformation visco-elasticity models including Mullins effect have been developed for doublenetwork hydrogels $[44,47,79]$. A fractional time power-dependent visco-elastic model for hydrogels has been developed by Keshavarz and co-workers [35], a more generic fluid-solid mechanical-based model by Birgersson and co-workers $[9,37]$ and a model which also includes poroelasticity has been developed by Wang and Wong [70].

An interesting alternative to the double-network hydrogels is doubly cross-linked, single-network hydrogels simultaneously containing both chemical and physical cross-links [11,13-15,22,24,34,39-43,48-51,53,58, $69,74,78,80-83]$ and, similarly, both strong and weak physical cross-links [14,27,45,60,64,65]. An attractive observation by e.g. Czarnecki et al. [15] is that it is possible to tune the strength of the physical cross-links by selecting a proper metal ion to modify the kinetics and thermodynamics of the adhesion-deadhesion activity of the physical cross-links resulting in a very broad potential relaxation time range of the temporary physical cross-links. This in turn results in a broad frequency range where within it is possible to tune the angular frequency $\omega_{\text {ald }}$ for maximum loss shear modulus $\max (\mathfrak{\Im} \hat{\mu})$ of the hydrogel, where $\hat{\mu}$ is the shear modulus, $\mathfrak{J}$ is the imaginary part, and subscripts a and $\mathrm{d}$ stand for the adhesion and deadhesion of the physical cross-links, respectively. For angular excitation frequencies well below $\omega_{\mathrm{a} \mid \mathrm{d}}$, the time is sufficiently long for the physical cross-links to debond (deadhesion) and for angular excitation frequencies well above $\omega_{\mathrm{a} \mid \mathrm{d}}$, the time is too short for the physical cross-links to debond; that is, almost only chemical cross-links of the hydrogels are active in the former case, while both chemical and physical cross-links are active in the latter case, see Fig. $1[15,49,58]$.

Stress-strain models for doubly cross-linked single-network hydrogels have been developed using finite deformation and fractional time power dependence [49], finite deformation and survivability functions using several material parameters [21,41-43], generalized Stokes-Einstein equation [50] and are reviewed by Creton [14] and Zhou et al. [82]. Of particular interest is the observed associative Rouse mode behaviour [59] of the contributing physical cross-link network part to the total shear modulus of the hydrogels showing a corresponding relaxation function with a time dependence of fractional order $-1 / 2$ and equivalently a shear modulus with a frequency dependence of fractional order $1 / 2[42,43,45,49]$. In this paper and based on the

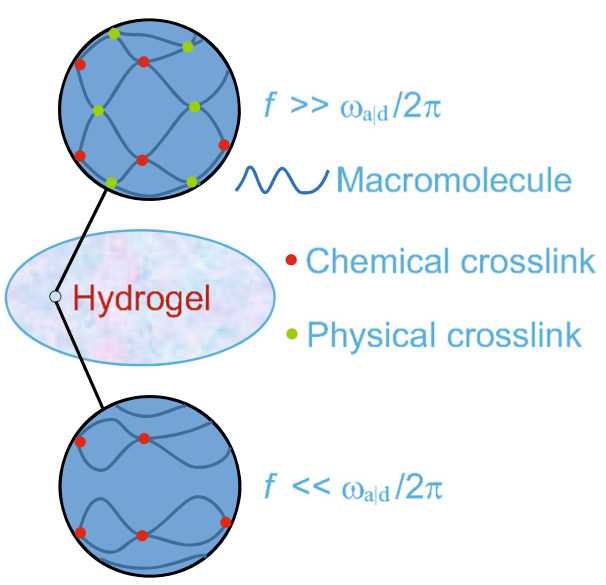

Fig. 1 Doubly cross-linked, single-network hydrogel, excited at low and high frequencies $f=\omega / 2 \pi$ 
latter observation, a visco-elastic model is derived by assuming nearly incompressible doubly cross-linked hydrogels with elastic spherical response and visco-elastic deviatoric response, using a Mittag-Leffler relaxation function [36] of order $1 / 2$ where generalized relaxation time is tunable, thus resulting in a tunable frequency $\omega_{\mathrm{a} \mid \mathrm{d}}$ for maximum loss shear modulus, in addition to a tunable equilibrium elastic modulus and relaxation intensity. It is possible to additively split the resulting fractional derivative-based shear modulus model into chemical and physical cross-link parts. Fractional calculus has found a broad application area including visco-elasticity, see e.g. Refs. [3-5,10,17,29,30,33,36,54-56,61,62,66-68,71], generally displaying a close agreement with those of measurements over a broad frequency range, in chemical and physical ageing modelling of elastomers [31,32] and in other fields, as shown in Machado et al. [46]. The derived model results are compared to those of measurements of doubly cross-linked, single-network polyvinyl alcohol (PVA) hydrogels of two different borax concentrations from Refs. [49,78]. Finally, the model is refined by additively including also an Abel relaxation function of order 1/2 into the deviatoric convolution integral to take into account the superimposed Rouse-type behaviour found in the measurements of the chemical cross-links in addition to their dominating elastic response. The simple, effective visco-elastic models developed are suitable in predicting the mechanical properties of tough, doubly cross-linked, single-network polyvinyl alcohol (PVA) hydrogels, including the separate chemical and physical cross-link contributions, with application potentials in tissue engineering and, more recently, as vibration isolators and dampers.

\section{Model}

The hydrogels are assumed homogeneous, isotropic, non-ageing and nearly incompressible with deformation confined to infinitesimal strain. The total second-order stress tensor $\sigma$ is additively decomposed into an elastic spherical part

$$
\operatorname{tr}[\sigma]=3 a \mu_{\infty} \operatorname{div}[\mathbf{u}],
$$

and a visco-elastic deviatoric part

$$
\operatorname{dev}[\sigma]=2 \mu_{\infty}\left\{\operatorname{dev}[\nabla \mathbf{u}]+\int_{-\infty}^{t} \Delta E_{\alpha}\left(-\left[\frac{t-s}{\tau_{\mathrm{rel}}}\right]^{\alpha}\right) \frac{\partial \operatorname{dev}[\nabla \mathbf{u}(s)]}{\partial s} \mathrm{~d} s\right\},
$$

respectively, using a convolution integral with a kernel of fractional standard linear solid[36], such as

$$
\boldsymbol{\sigma}=\frac{1}{3} \operatorname{tr}[\boldsymbol{\sigma}] \mathbf{i}+\operatorname{dev}[\boldsymbol{\sigma}],
$$

where $\mathbf{u}$ is first-order displacement tensor, $t$ is time, $a \gg 1$ is a nearly incompressible material constant (with $a \rightarrow \infty$ being the incompressible limit), $\mu_{\infty}$ is the equilibrium elastic modulus, and $\Delta$ is a non-dimensional relaxation intensity,

$$
E_{\alpha}(x)=\sum_{n=0}^{\infty} \frac{x^{n}}{\Gamma(1+\alpha n)},
$$

is the Mittag-Leffler function, $\Gamma(z)=\int_{0}^{\infty} s^{z-1} e^{-s} \mathrm{~d} s, z>0$, is the Gamma function, $\tau_{\text {rel }}$ is generalized relaxation time, $0<\alpha<1$ is a material constant, $\mathbf{i}$ is second-order unit tensor, $\nabla$ is the nabla operator, and the operators $\operatorname{tr}[\cdot] \operatorname{div}[\cdot]$ and $\operatorname{dev}[\cdot]$ denote trace, divergence and deviator, respectively.

Based on the observed associative Rouse mode behaviour [59] of the contributing physical cross-link network displaying a relaxation function with a time dependence of fractional order $-1 / 2$ and equivalently a shear modulus with a frequency dependence of fractional order $1 / 2[42,45,49]$, the material constant for the hydrogel is set to $\alpha=1 / 2$ being a generalization of the associative Rouse mode behaviour displaying a relaxation function with an approximative time dependence of fractional order $-1 / 2$ in the long time range and shown below. The visco-elastic deviatoric stress Eq. (2) becomes

$$
\operatorname{dev}[\boldsymbol{\sigma}]=2 \mu_{\infty}\left\{\operatorname{dev}[\nabla \mathbf{u}]+\int_{-\infty}^{t} \Delta E_{1 / 2}\left(-\sqrt{\frac{t-s}{\tau_{\text {rel }}}}\right) \frac{\partial \operatorname{dev}[\nabla \mathbf{u}(s)]}{\partial s} \mathrm{~d} s\right\}
$$

which is possible to reformulate [26] as

$$
\operatorname{dev}[\boldsymbol{\sigma}]=2 \mu_{\infty}\left\{\operatorname{dev}[\nabla \mathbf{u}]+\int_{-\infty}^{t} \Delta \operatorname{erfcx}\left(\sqrt{\frac{t-s}{\tau_{\text {rel }}}}\right) \frac{\partial \operatorname{dev}[\nabla \mathbf{u}(s)]}{\partial s} \mathrm{~d} s\right\},
$$


where the scaled complementary error function

$$
\operatorname{erfcx}(z)=\frac{2 \mathrm{e}^{z^{2}}}{\sqrt{\pi}} \int_{z}^{\infty} \mathrm{e}^{-s^{2}} \mathrm{~d} s=\mathrm{e}^{z^{2}}[1-\operatorname{erf}(z)],
$$

being a numerically stable function reducing possible under- and overflow during its numerical estimation, and the error function

$$
\operatorname{erf}(z)=\frac{2}{\sqrt{\pi}} \int_{0}^{z} \mathrm{e}^{-s^{2}} \mathrm{~d} s .
$$

Using an asymptotic expansion of the relaxation function [57], that is, of the scaled complementary error function, results in

$$
\operatorname{erfcx} \sqrt{t^{*}} \sim \frac{1}{\sqrt{\pi t^{*}}},
$$

as $t^{*} \rightarrow \infty$ where normalized time $t^{*}=t / \tau_{\text {rel }}$. As a result, the relaxation function for the hydrogels displays an approximative normalized time dependence of $1 / \sqrt{t^{*}}$ as $t^{*} \rightarrow \infty$, that is, of a fractional order $-1 / 2$ in the long time range while reducing to that of the observed associative Rouse mode behaviour [59]. In passing, it is noted that e.g. Bagley and Torvik [4] and Wharmby and Bagley [71] apply half-order derivatives in their stressstrain relations, while identifying the Rouse mode behaviour, being the long time/low-frequency asymptote of the present model. Furthermore, the long normalized time approximation of the present relaxation function is identical to the Abel operator kernel [36]

$$
\operatorname{erfcx} \sqrt{t^{*}} \approx I_{1 / 2}\left(t^{*}\right)=\frac{1}{\Gamma\left(\frac{1}{2}\right) \sqrt{t^{*}}},
$$

as $t^{*} \rightarrow \infty$, where the specific value of the Gamma function $\Gamma(0.5)=\sqrt{\pi}$. On the other hand, the relaxation function displays [57]

$$
\operatorname{erfcx} \sqrt{t^{*}} \approx 1-2 \sqrt{\frac{t^{*}}{\pi}} \sim \exp \left(-\frac{\sqrt{t^{*}}}{\Gamma\left(\frac{3}{2}\right)}\right),
$$

as $t^{*} \rightarrow 0^{+}$, where $\Gamma(1.5)=\sqrt{\pi} / 2$; that is, the relaxation function at a short normalized time shows a stretched exponential function behaviour with a very fast decay at increasing normalized time.

Temporal Fourier transformations $[\stackrel{\cdot}{\cdot}]=\int_{-\infty}^{\infty}[\cdot] \exp (-\mathrm{i} \omega t) \mathrm{d} t$ of the constitutive Eqs. (1) and (5), where $\mathrm{i}$ is the imaginary unit, result in

$$
\operatorname{tr}[\widetilde{\boldsymbol{\sigma}}]=3 a \mu_{\infty} \operatorname{div}[\widetilde{\mathbf{u}}]
$$

and

$$
\operatorname{dev}[\widetilde{\boldsymbol{\sigma}}]=2 \mu_{\infty}\left\{1+\frac{\Delta \sqrt{\mathrm{i} \omega^{*}}}{1+\sqrt{\mathrm{i} \omega^{*}}}\right\} \operatorname{dev}[\nabla \widetilde{\mathbf{u}}],
$$

where the normalized angular frequency $\omega^{*}=\omega / \omega_{\mathrm{a} \mid \mathrm{d}}$ and the material parameter $\omega_{\mathrm{a} \mid \mathrm{d}}$ is related to the generalized relaxation time as $\omega_{\mathrm{a} \mid \mathrm{d}}=1 / \tau_{\text {rel }}$. It should be noted, as stated in the Introduction section, that fractional derivative models have been applied previously also for visco-elasticity. It is the additive split in chemical and physical cross-link contributions that is the study focus. This is considered next.

The shear modulus $\hat{\mu}$ defined via $\operatorname{dev}[\widetilde{\boldsymbol{\sigma}}]=2 \hat{\mu} \operatorname{dev}[\nabla \widetilde{\mathbf{u}}]$ in Eq. (13) is possible to additively split into

$$
\hat{\mu}=\hat{\mu}_{\mathrm{Ch}}+\hat{\mu}_{\mathrm{Ph}},
$$

where $\hat{\mu}_{\mathrm{Ch}}$ and $\hat{\mu}_{\mathrm{Ph}}$ are the shear modulus derived from chemical and physical cross-links, respectively, see e.g. Refs. $[15,49]$. In particular, the normalized shear modulus $\hat{\mu}^{*}=\hat{\mu} / \mu_{\infty}$ with $\hat{\mu}$ from Eq. (13) displays

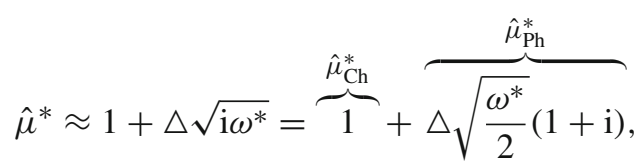

for $\omega^{*} \ll 1$, while using $\sqrt{\mathrm{i}}=(1+\mathrm{i}) / \sqrt{2}$. That is, the normalized shear modulus derived from the chemical cross-links is approximately normalized frequency independent and elastic (only showing a non-vanishing 
normalized storage modulus), while the normalized shear modulus derived from corresponding physical crosslinks displays an identical normalized storage and loss modulus

$$
\Re \hat{\mu}_{\mathrm{Ph}}^{*}=\Im \hat{\mu}_{\mathrm{Ph}}^{*}=\Delta \sqrt{\frac{\omega^{*}}{2}},
$$

for $\omega^{*} \ll 1$, where $\Re$ is the real part, being similar to associative Rouse mode behaviour [59] and other results $[42,45,49]$, however, and most importantly, here with the coefficient in front of $\sqrt{\omega^{*}}$ (in Eq. (16)) given in terms of physically quantifying material parameter $\triangle$. Furthermore, the maximum normalized loss modulus

$$
\max \left\{\mathfrak{\Im} \hat{\mu}^{*}\right\}=\frac{\triangle}{2(1+\sqrt{2})},
$$

at an angular frequency for maximum dissipation $\omega^{*}=1$ due to the maximum adhesion-deadhesion activity of the physical cross-links. In passing, it may be noted that the maximum normalized loss modulus max $\left\{\Im \hat{\mu}_{\mathrm{Ph}}^{*}\right\}$ derived from the physical cross-links only and its normalized angular frequency locus are identical to those of the corresponding hydrogel $\hat{\mu}^{*}$ derived from both chemical and physical cross-links due to the model applied, displaying a purely elastic, frequency-independent normalized shear modulus $\hat{\mu}_{\mathrm{Ch}}^{*}$ derived from the chemical cross-links only and being identical to its normalized storage modulus $\Re \hat{\mu}_{\mathrm{Ch}}^{*}$ and to the normalized equilibrium elastic modulus $\mu_{\infty}^{*} \equiv 1$. Finally, the normalized shear modulus from Eq. (13) displays

$$
\hat{\mu}^{*} \approx 1+\triangle,
$$

for $\omega^{*} \gg 1$. That is, the normalized shear modulus is showing an elastic, normalized frequency-independent behaviour being identical to the normalized storage modulus with vanishing loss modulus where relaxation intensity $\triangle$ may physically be identified as the relative contribution of the physical cross-links at full activity to the normalized total shear modulus in relation to the corresponding contribution of the chemical cross-links. A simple estimation example of the relaxation intensity is $\Delta=v_{\mathrm{Ph}} / \nu_{\mathrm{Ch}}$ for an ideally, isotropic, doubly and tetrafunctionally cross-linked, single network with equal-functional and homogeneous distribution of chemical and physical cross-links, lacking any trapped entanglements, where $v_{\mathrm{Ch}}$ and $\nu_{\mathrm{Ph}}$ are the cross-link densities in mol per volume for chemical and maximally active physical cross-links, respectively [15]. Furthermore, simple and possible physical models to estimate the equilibrium elastic modulus include, but are not limited to, affine and phantom network models [16], reading $\mu_{\infty}^{\text {affine }}=\chi v_{\mathrm{Ch}} R T / 2$ and $\mu_{\infty}^{\text {phantom }}=[1-2 / \chi] \chi v_{\mathrm{Ch}} R T / 2$, respectively, where $R=8.314 \mathrm{~J} / \mathrm{molK}$ is the universal molar gas constant, $T$ is temperature, and $\chi$ is cross-link functionality, that is, the number of chain segments emanating from each chemical cross-link, being $\chi=4$ for tetra-functional cross-link networks. Physically, the increasing density of active physical cross-links at increasing normalized frequency and, thus, decreasing time for debonding of physical cross-links result in an increased total cross-link density, including both chemical and physical cross-links. This in turn results in a reduced network flexibility and reduced conformal freedom which in turn result in an entropy governed increase of the normalized storage modulus of the doubly cross-linked, single-network hydrogel [15].

Finally, the normalized shear modulus from Eq. (13), valid throughout the whole normalized frequency range, splits additively into

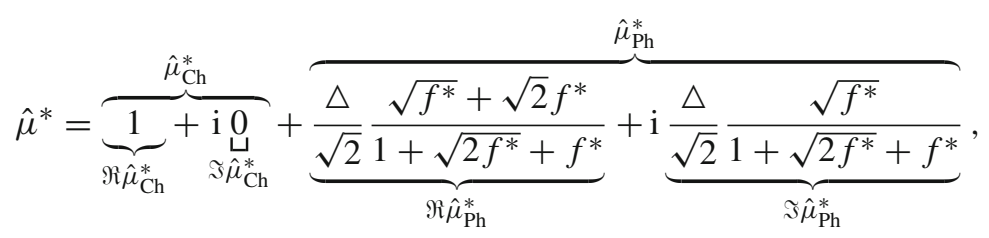

where the chemical and physical parts from Eq. (14) are explicitly given and the normalized frequency $f^{*}=$ $f / f_{\mathrm{a} \mid \mathrm{d}}$. Note that the relation between the frequency $f$ and the angular frequency $\omega$ is $2 \pi f=\omega$, while the normalized relation reads $f^{*}=\omega^{*}$. Note also that the normalized loss modulus $\Im \hat{\mu}_{\mathrm{Ch}}^{*}$ derived from the chemical cross-links only is assumed to vanish. This is a plausible assumption as in general $\Im \hat{\mu}_{\mathrm{Ch}}^{*} \ll \Im \hat{\mu}_{\mathrm{Ph}}^{*}$ for real doubly cross-linked hydrogels, see e.g. Refs. [15,49,58,78].

The normalized relaxation spectrum $\gamma^{*}$ to the normalized relaxation functions in Eq. (6) is defined via

$$
\triangle \operatorname{erfcx} \sqrt{t^{*}}=\int_{\tau^{*}=0^{-}}^{\infty} \gamma^{*}\left(\tau^{*}\right) \mathrm{e}^{-t^{*} / \tau^{*}} \mathrm{~d}\left\{\log _{\mathrm{e}}\left(\tau^{*}\right)\right\},
$$


for $t^{*} \geq 0$, respectively, where $\tau^{*}=\tau / \tau_{\text {rel }}$ is the normalized relaxation time. The normalized relaxation spectrum is possible to obtain from the normalized shear modulus [20] as

$$
\gamma^{*}\left(\tau^{*}\right)=\frac{1}{\pi} \Im\left\{\lim _{\epsilon \rightarrow 0^{+}} \breve{\mu}^{*}\left(-\frac{1}{\tau^{*}}+\mathrm{i} \epsilon\right)\right\},
$$

where $\breve{\mu}^{*}\left(i \omega^{*}\right) \equiv \widehat{\mu}^{*}\left(\omega^{*}\right)$, resulting in

$$
\gamma^{*}\left(\tau^{*}\right)=\frac{\triangle}{\pi} \frac{\sqrt{\tau^{*}}}{1+\tau^{*}}
$$

Obviously, the normalized spectrum is continuous while displaying a maximum of

$$
\max \left\{\gamma^{*}\right\}=\frac{\triangle}{2 \pi},
$$

located at the normalized relaxation time $\tau^{*}=1$. Clearly, the generalized relaxation time $\tau_{\text {rel }}$ applied in the constitutive Eq. (6) is the relaxation time for maximum relaxation spectrum. Spectrum is useful while investigating visco-elasticity and molecular motion, being intelligible as the specific molecular time reaction range, involving broad time scales, thus making a natural logarithmic scale (20) most appropriate. Clearly, the generalized relaxation time for hydrogel is physically interpreted as the optimal relaxation time for adhesiondeadhesion of physical cross-links [58].

\section{Results and discussion}

The normalized shear storage and loss modulus with $\Delta=10$ are shown in Fig. 2 versus the normalized frequency together with the loss factor, determined as

$$
\eta=\frac{\Im\left[\hat{\mu}_{\mathrm{Ch}}+\hat{\mu}_{\mathrm{Ph}}\right]}{\Re\left[\hat{\mu}_{\mathrm{Ch}}+\hat{\mu}_{\mathrm{Ph}}\right]} .
$$

Clearly, the studied hydrogel is essentially displaying a fast normalized storage modulus increase with the normalized frequency, starting at a low-frequency normalized storage modulus plateau of about 1 and is clearly approaching a high normalized frequency plateau of $1+\Delta=11$ at the high normalized frequency end; the latter observations are in accordance with the high normalized frequency approximation (18). The normalized

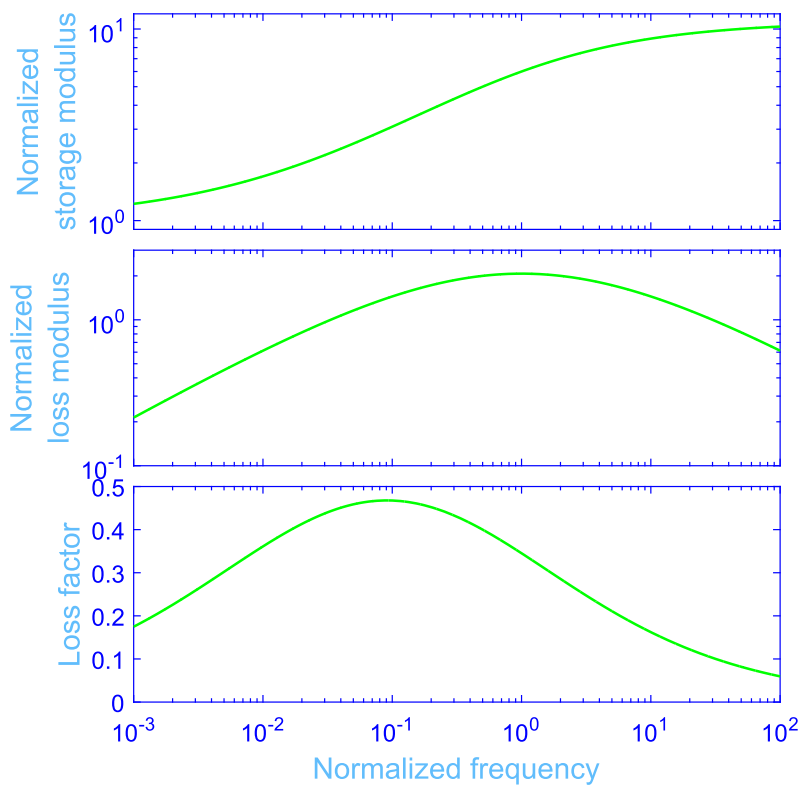

Fig. 2 Normalized storage, loss modulus and loss factor versus normalized frequency with $\triangle=10$ 
loss modulus is peaking at $f^{*}=1$, not surprising, as the hydrogel is tuned to peak at that normalized frequency while displaying a maximum normalized loss modulus of $\triangle /(2+2 \sqrt{2})=2.07$, in accordance with Eq. (17). The maximum loss factor of the studied hydrogels is

$$
\max (\eta)=\frac{\triangle}{[\sqrt{2}+\sqrt{1+\triangle}]^{2}-1}=0.47,
$$

that is $47 \%$, while peaking at a lower normalized frequency than the corresponding normalized frequency for maximum normalized loss modulus, namely at $f^{*}=1 /[1+\Delta]=0.09$.

A detailed study over the normalized moduli versus the normalized frequency with $\Delta=10$ is shown in Fig. 3 while using Eq. (19), displaying the contributions from the chemical and physical cross-links and their normalized frequency dependencies. Clearly, the normalized storage modulus due to the contributions from the chemical cross-links only is displaying a normalized frequency-independent behaviour while fully generating the normalized total storage modulus at vanishing normalized frequency. In contrast, the normalized storage modulus due to the contributions from the physical cross-links only disappears at vanishing normalized frequency while showing a normalized frequency dependence of fractional order $1 / 2$ in the low normalized frequency range. Additionally and for enhanced visual clarity, a function with a normalized frequency dependence of fractional order $1 / 2$ is depicted in Fig. 3. Furthermore, the normalized storage modulus due to the contributions from the physical cross-links only coincides with its normalized loss modulus in the low normalized frequency range, in accordance with the preceding research results [49], being similar to the associated Rouse mode behaviour[59]. A conceivably ingenuous physical explanation of the dynamic behaviour of a doubly cross-linked single-network hydrogel over the normalized frequency range is depicted within the circles in Fig. 3, displaying the hydrogel consisting of chemically and physically cross-linked macromolecules abundantly swollen with water, while being evaluated at various normalized frequencies. At a very low normalized frequency $\left(f^{*} \ll 0.001\right)$, almost at a quasi-static state, the period time of the mechanical oscillation is very long and all temporary physical cross-links are debonded (after bonding) within a time frame much less than the period time of the mechanical oscillation. Consequently, the influence of the temporary physical cross-links is negligible within the period time of the mechanical oscillation and only the chemical cross-links

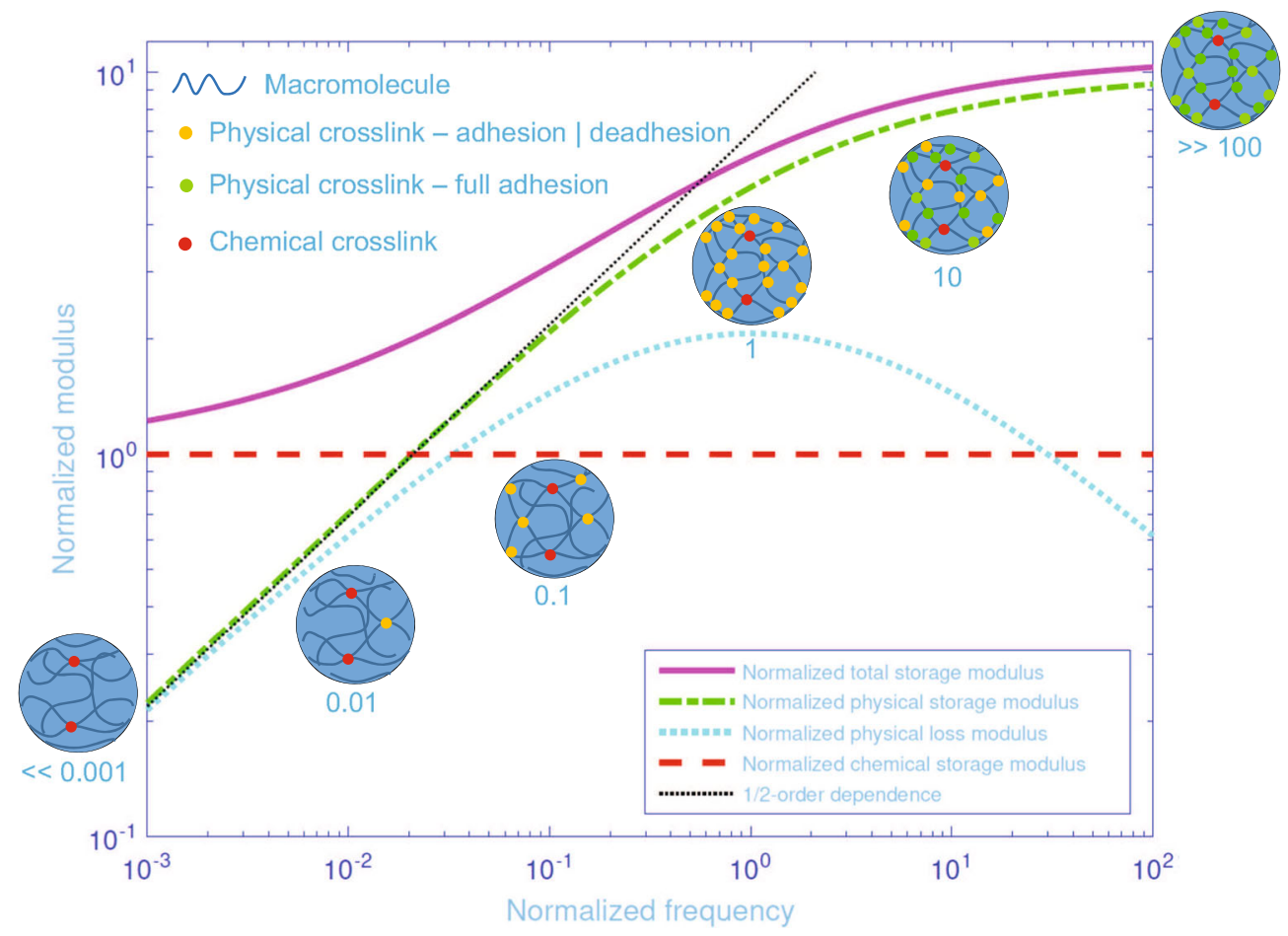

Fig. 3 Normalized moduli of a doubly cross-linked, single-network hydrogel versus normalized frequency with $\triangle=10$, together with a symbolical indication of chemical and physical cross-link activity in circles 
are active, symbolically indicated by the two red $\bullet$ chemical cross-links in the circle above $\ll 0.001$ in Fig. 3 . The hydrogel is displaying essentially an elastic behaviour within this very low normalized frequency range with the normalized storage modulus mainly proportional to the chemical cross-link density. At increasing normalized frequency, the influence of the temporary physical cross-links increases; they still have time to debond (after bonding), though the period time of the mechanical oscillations is not any longer very long compared to all the temporary physical adhesion-deadhesion processes. That is, the physical cross-links start to be active while simultaneously contributing to the normalized storage modulus by the increased total effective cross-link density and to the normalized loss modulus by the dissipation activity connected to the adhesion-deadhesion operations of the physical cross-links. This is a plausible physical explanation of the simultaneous increase of the normalized storage and loss modulus with the increased normalized frequency; the higher the normalized frequency is, the larger is the increase due to the shortened mechanical oscillation time while approaching that of the time frame for adhesion-deadhesion activity of the physical cross-links. This results in a relatively more active physical cross-links within the period time of the mechanical oscillation, symbolically indicated by the one, five and the twenty yellow $\bullet$ physical cross-links undergoing adhesion and deadhesion operations in the circles above $0.01,0.1$ and 1, respectively, in Fig. 3. At $f^{*}=1$, the period time of the mechanical oscillation matches perfectly the time frame for adhesion-deadhesion activity of the physical cross-links, resulting in an optimum dissipation and, thus, a maximum normalized loss modulus with maximum adhesion and deadhesion active physical cross-links within the period time of mechanical oscillation, symbolically indicated by the twenty yellow $\bullet$ physical cross-links undergoing adhesion and deadhesion operations in the circle above 1 in Fig. 3. At further increased normalized frequency, the period time of the mechanical oscillation is shorter than the time frame for adhesion-deadhesion activity of the physical cross-links, resulting in physical crosslinks that do not have sufficient time to debond (after bonding), thus resulting in a decreased normalized loss modulus due to the decreased adhesion-deadhesion activity of the physical cross-links, in addition to the increased normalized storage modulus due to the increased total effective cross-link density. The higher the normalized frequency is, the shorter is the period time of the mechanical oscillation and, thus, the higher is the activity of the non-debonding physical cross-links, symbolically indicated by the twelve and twenty green • non-debonding physical cross-links in the circles above 10 and $\gg 100$, respectively, in Fig. 3. At a very high frequency, $f^{*} \gg 100$, the period time for the mechanical oscillation is very short and all possible temporary physical cross-links are non-debonding, symbolically indicated by the twenty green $\bullet$ non-debonding physical cross-links in the circle above $\gg 100$. This results in a high normalized storage modulus, being proportional to the total chemical and physical cross-link density, symbolically indicated by the two red $\bullet$ chemical crosslinks plus the twenty green $\bullet$ non-debonding physical cross-links in the circles above $\gg 100$. However, the normalized loss modulus is vanishing due to the disappearing adhesion-deadhesion activity of the physical cross-links, symbolically indicated by the vanishing number of yellow • physical cross-links undergoing adhesion and deadhesion operations in the circle above $\gg 100$. The normalized storage modulus at a very high normalized frequency is about $11(1+\Delta)$ times that of the corresponding normalized storage modulus at vanishing normalized frequency, achievable by selecting a maximum physical cross-link density ten times higher than the corresponding chemical cross-link density, symbolically indicated by the ratio of twenty green - non-debonding physical cross-links to two red $\bullet$ chemical cross-links, that is $20 / 2=10$, in the circles above $\gg 100$. Although the normalized storage modulus due to the contributions from the physical cross-links only disappears at vanishing normalized frequency and the normalized frequency for the maximum normalized loss modulus is as high as $f^{*}=1$, the normalized frequency for equal contribution from the physical and chemical cross-links to the normalized storage modulus is nevertheless very low, only about $f^{*}=0.0207$. The main reason is the relatively high physical cross-link density compared to the corresponding chemical cross-link density, resulting in a fast increase of the normalized storage modulus with increased normalized frequency due to a relatively fast growing contribution from the physical cross-links. In passing, it is noted that other dissipation mechanisms may exist although adhesion-deadhesion activity of the physical cross-links prevails over those alterative mechanisms, thus making a purely elastic normalized storage modulus due to the contributions from the chemical cross-links only and with vanishing normalized loss modulus, a suitable model $[15,58]$. Finally, similar physical explanations as above are given elsewhere $[15,49,58]$, although the physical explanations, nevertheless, in this study are comprehensively clarified.

The normalized relaxation spectrum $\gamma^{*}$ with $\Delta=10$ is shown in Fig. 4 versus the normalized relaxation time $\tau^{*}$, ranging over a very broad normalized time scale. Clearly, the normalized relaxation time locus for the maximum normalized relaxation spectrum is $\tau^{*}=1$ while displaying a maximum normalized relaxation spectrum of 1.59. Physically, the generalized relaxation time for hydrogel is of the same order as the optimal time frame for adhesion-deadhesion process of physical cross-links [58]. Indeed, the optimal time frame is 


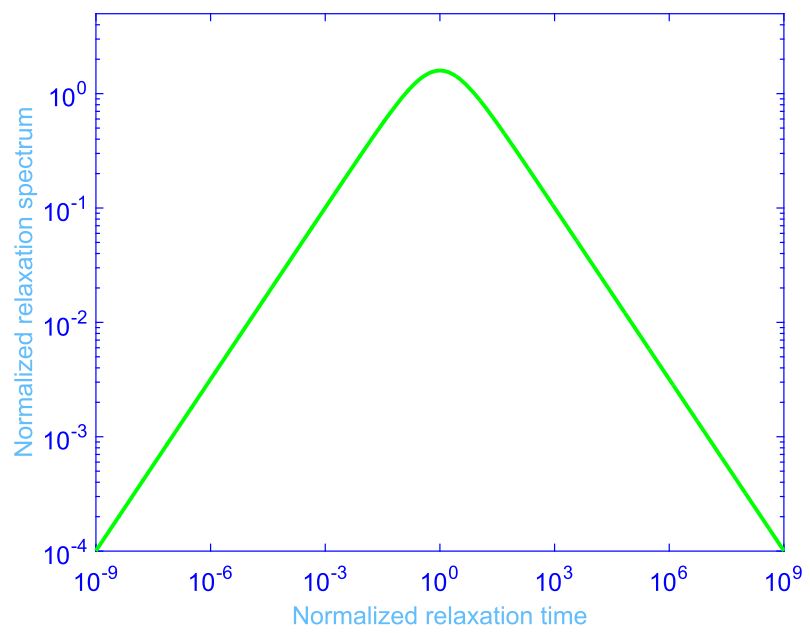

Fig. 4 Normalized relaxation spectrum versus normalized relaxation time with $\Delta=10$

$T_{\mathrm{opt}} \equiv 1 / f_{\mathrm{a} \mid \mathrm{d}}=2 \pi \tau_{\mathrm{rel}}$, derived from the constitutive material parameter relation $\omega_{\mathrm{a} \mid \mathrm{d}}=1 / \tau_{\text {rel }}$, reading in normalized form $T_{\mathrm{opt}}^{*} \equiv T_{\mathrm{opt}} / \tau_{\mathrm{rel}}=2 \pi$. Furthermore, the relaxation spectrum in Fig. 4 is continuous, thus enabling a wider time scale most likely found in the real molecular networks and in the adhesion-deadhesion physical cross-links processes to be successfully taken into account by the applied Mittag-Leffler and the scaled complementary error relaxation function, while additionally giving a richer structure to the model.

Finally, the model results are compared to those of the measurements from Mayumi et al. [49] and Zhao et al. [78]. The doubly cross-linked, single-network hydrogels consist of poly(vinyl alcohol) (PVA) chemically cross-linked in an aqueous solutions with glutaraldehyde (GA) at a PVA concentration of $12 \mathrm{wt} \%$ and a GA concentration of $5.5 \mathrm{mM}$ corresponding to $0.2 \mathrm{~mol} \%$ of GA to monomer unit of PVA. The physical cross-links are borate ions acting as transient cross-links between the PVA chains and prepared by immersing the chemically cross-linked PVA hydrogels into an aqueous solutions of borax and $\mathrm{NaCl}$ of concentrations of $1 \mathrm{mM} \mathrm{[49],}$ $50 \mathrm{mM}$ [78] and $90 \mathrm{mM}$ [49], $460 \mathrm{mM}$ [78], respectively, corresponding to $1.5 \mathrm{~mol} \%$ [49] and $73 \mathrm{~mol} \%$ [78], respectively, of borate to monomer unit of PVA. The reader is referred to Mayumi et al. [49] and Zhao et al. [78] for the details regarding the sample preparations and measurements of the various shear moduli. The results are in Fig. 5 showing the normalized total storage modulus, physical storage modulus, physical loss modulus, chemical storage modulus and the normalized chemical loss modulus versus the normalized frequency $f^{*}$, for the model where $\Delta^{(\mathrm{a})}=12.3$ and $\Delta^{(\mathrm{b})}=28.4$, respectively, and for the measurements [49] ${ }^{(\mathrm{a})},[78]^{(\mathrm{b})}$, however, displaying a courser measurement normalized frequency mesh than those in Mayumi et al. (Fig. 2) [49] and Zhao et al. (Figs. 5a and 10a) [78] for visual clarity. Other materials parameters applied read $\mu_{\infty}^{(a)}=1500 \mathrm{~N} / \mathrm{m}^{2}$, $\mu_{\infty}^{(\mathrm{b})}=1700 \mathrm{~N} / \mathrm{m}^{2}$ and $f_{\mathrm{a} \mid \mathrm{d}}^{(\mathrm{a})}=0.6 / 2 \pi \mathrm{Hz}, f_{\mathrm{a} \mid \mathrm{d}}^{\text {(b) }}=5.0 / 2 \pi \mathrm{Hz}$, respectively. Clearly, the model results and those of the measurements match remarkably well over the broad normalized frequency ranges while using only three physically quantifiable material parameters: the frequency for maximum loss modulus, the equilibrium elastic modulus and the relaxation intensity. In particular, the model results and those of the measurements of the normalized total and physical storage modulus almost overlap each other, the normalized loss modulus displays identical pattern although differing slightly more in Fig. 5a, while almost matching in Fig. 5b. Moreover, the elastic, normalized frequency-independent chemical storage modulus is clearly a suitable model displaying an almost perfect overlap between measurement results and those of the model over the normalized frequency range with a small deviation at the higher normalized frequency range. Furthermore, the normalized chemical loss modulus measurement results display $\Im \hat{\mu}_{\mathrm{Ch}}^{*} \ll \Im \hat{\mu}_{\mathrm{Ph}}^{*}$ throughout the normalized frequency range, thus making a vanishing normalized chemical loss modulus $\Im \hat{\mu}_{\mathrm{Ch}}^{*} \approx 0$ a suitable model. Finally, the shear modulus (19) was generalized to allow for other $\alpha$ values than $1 / 2$ via temporal Fourier transform of Eq. (2) while subsequently minimizing the relative least square difference between the measured $\hat{\mu}_{n}^{* \text { meas }}$ and modelled $\hat{\mu}_{n}^{* \bmod }(\alpha)$ normalized shear modulus

$$
\min _{0<\alpha<1} \sum_{n=1}^{n_{\text {tot }}}\left|\frac{\hat{\mu}_{n}^{* \text { meas }}-\hat{\mu}_{n}^{* \text { mod }}(\alpha)}{\hat{\mu}_{n}^{* \text { meas }}}\right|^{2},
$$



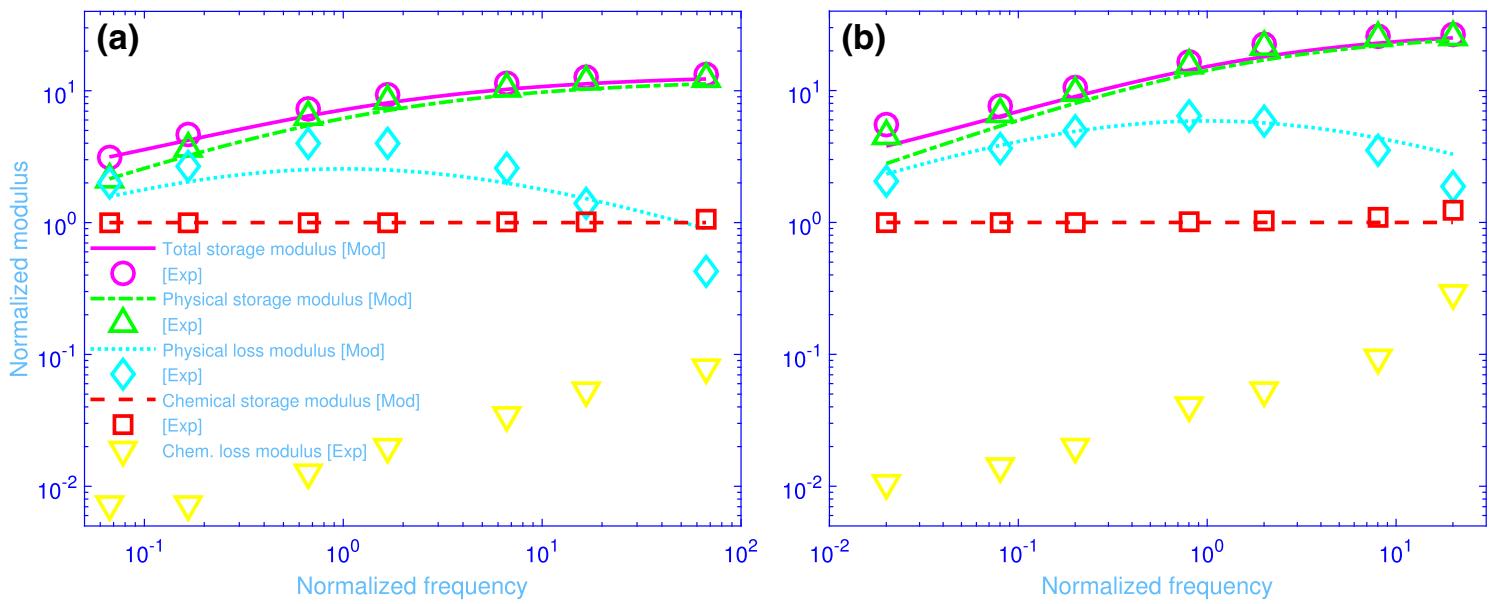

Fig. 5 Normalized moduli of a doubly cross-linked, single-network poly(vinyl alcohol) (PVA) hydrogel with glutaraldehyde (GA) chemical cross-links concentration of $0.2 \mathrm{~mol} \%$ of GA to monomer unit of PVA, and borate ions physical cross-links, versus normalized frequency. a Model results (lines) with $\Delta=12.3$ compared to those of measurements (symbols) from Mayumi et al. (Fig. 2) [49] at physical cross-link concentration of $1.5 \mathrm{~mol} \%$ of borate to monomer unit of PVA. b Model results (lines) with $\triangle=28.4$ compared to those of measurements (symbols) from Zhao et al. (Figs. 5a and 10a) [78] at physical cross-link concentration of $73 \mathrm{~mol} \%$ of borate to monomer unit of PVA

by a MATLAB ${ }^{\circledR}$ constrained nonlinear multi-variable programming solver fmincon, where $n_{\text {tot }}$ is the total number of measurement points, resulting in an optimum $\alpha^{\text {optimum }}=0.55$ for the measurement in Fig. 5a. Consequently, the shear modulus (19) with $\alpha=1 / 2$ is an appropriate model while also showing a Rouse mode behaviour of the physical storage and loss modulus with the fractional half-order frequency dependence at low normalized frequencies. It should be noted that other models with more parameters such as 7-parameter [42,43] and 4-parameter [21] large strain models including survivability functions have been tested for doubly crosslinked, single-network PVA hydrogels also showing a good fit.

\subsection{Refined model}

It may be observed that the normalized chemical loss modulus measurement results in Fig. 5a, b also display a Rouse-type behaviour with a normalized frequency dependence of fractional order $1 / 2$ throughout the normalized frequency range and that the normalized chemical storage modulus measurement results display a slight increase and a small deviation from the constant value at the higher normalized frequency range. It is possible to extend the present 3-parameter model Eq. (5) into a 4-parameter model by additively including also an Abel relaxation function of order $1 / 2$ into the deviatoric convolution integral resulting in

$$
\begin{aligned}
\operatorname{dev}[\sigma]=2 \mu_{\infty}\{\operatorname{dev}[\nabla \mathbf{u}] \\
\left.+\int_{-\infty}^{t}\left[\mathcal{C} I_{1 / 2}\left(\frac{t-s}{\tau_{\text {rel }}}\right)+\Delta E_{1 / 2}\left(-\sqrt{\frac{t-s}{\tau_{\text {rel }}}}\right)\right] \frac{\partial \operatorname{dev}[\nabla \mathbf{u}(s)]}{\partial s} \mathrm{~d} s\right\},
\end{aligned}
$$

where the fourth parameter $\mathcal{C}$ may be named as a chemical Rouse stress intensity factor. The resulting normalized shear modulus, valid throughout the whole normalized frequency range, splits additively into

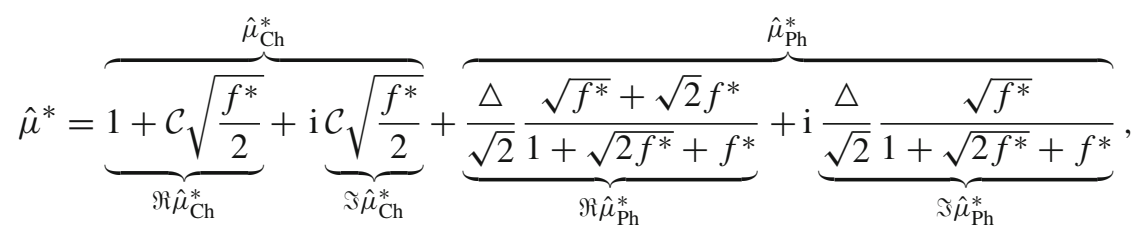

where the 4-parameters are the equilibrium elastic modulus, the frequency for maximum loss modulus, the relaxation intensity and the chemical Rouse stress intensity factor. The chemical half-order normalized frequency dependence in Eq. (28) is similar to that of e.g. Bagley and Torvik [4] and Wharmby and Bagley [71], 

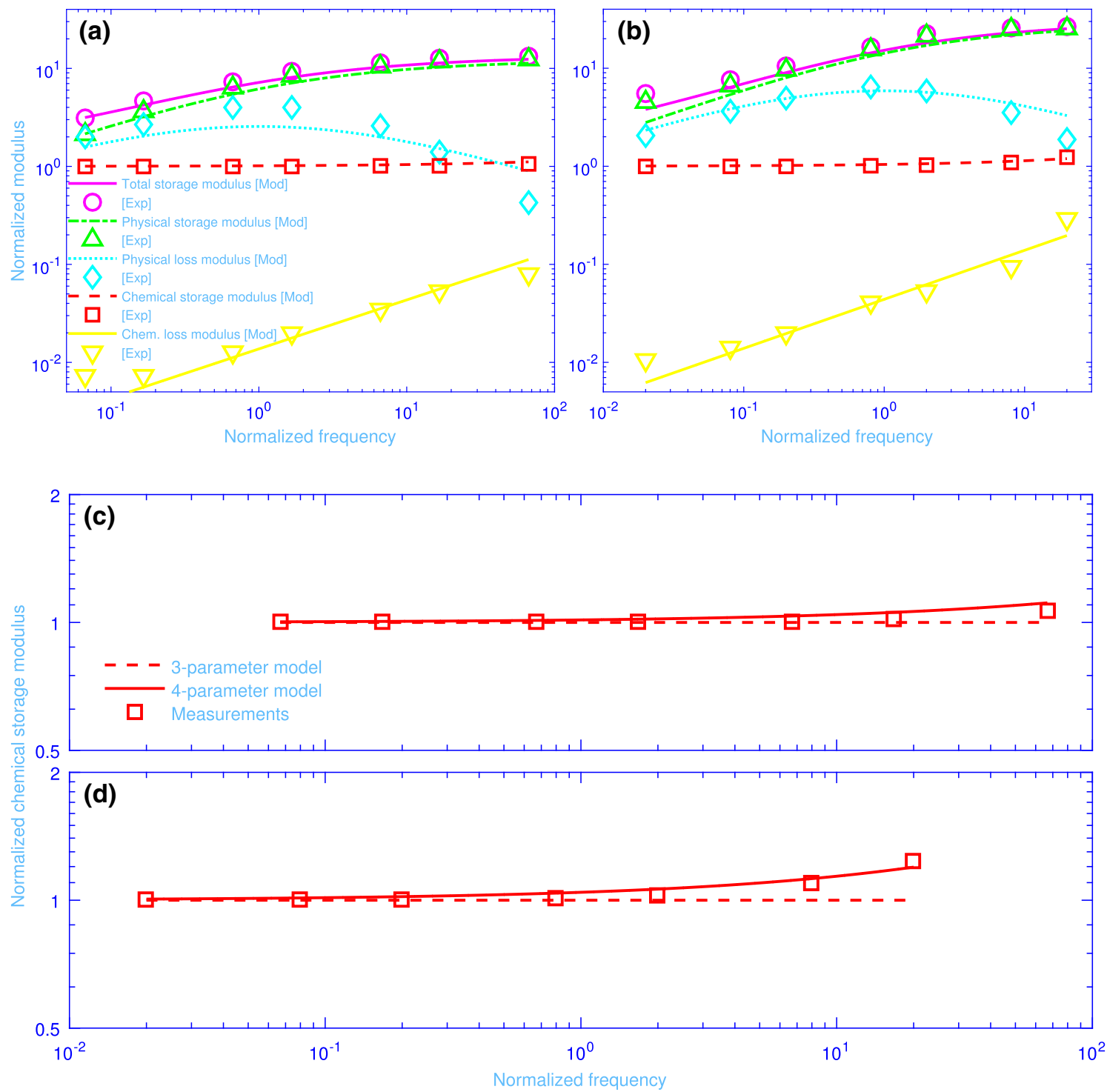

Fig. 6 The 4-parameter normalized modulus model results in $\mathbf{a}$ and $\mathbf{b}$ are compared to the same measurements results as in Fig. 5a, b from Mayumi et al. [49] ${ }^{(\mathrm{a})}$ and Zhao et al. [78 ${ }^{(\mathrm{b})}$, respectively, while using identical material parameter values as in Fig. 5a, b in addition to $\mathcal{C}^{(\mathrm{a})}=0.0194$ and $\mathcal{C}^{(\mathrm{b})}=0.0621$. The 3- and 4-parameter model results for normalized chemical storage modulus in $\mathbf{c}$ and $\mathbf{d}$ are compared to those of measurements from Fig. 5a, b, respectively

while they identified the Rouse mode behaviour in stress-strain relations. The 4-parameter model results are compared to the same measurements results as in Fig. 5a, b from Mayumi et al. [49] ${ }^{(\mathrm{a})}$ and Zhao et al. [78] ${ }^{(\mathrm{b})}$, respectively, while using the identical material parameter values as in Fig. $5 \mathrm{a}$, b, in addition to $\mathcal{C}^{(\mathrm{a})}=0.0194$ and $\mathcal{C}^{(\mathrm{b})}=0.0621$. The optimum chemical Rouse stress intensity factors are calculated by the MATLAB ${ }^{\circledR}$ constrained nonlinear multi-variable programming solver fmincon minimizing the relative least square difference between modelled and measured normalized chemical loss modulus. Clearly, the 4-parameter model results for the normalized chemical loss modulus and those of the measurements match well throughout the normalized frequency range in Fig. 6a, b. Moreover, the 4-parameter model results for normalized chemical storage modulus display a slight increase with normalized frequency in accordance with those of the measurements in Fig. 6a, b. Furthermore, the 3- and 4-parameter model results for the normalized chemical storage modulus in Fig. 6c, d are compared to those of the measurements from Fig. 5a, b, respectively. Clearly, the 4-parameter model results for the normalized chemical storage modulus match remarkably well with those of measurements. 


\section{Conclusions}

The derived visco-elastic 3-parameter model for doubly cross-linked, single-network polyvinyl alcohol hydrogels:

- Covers the low-frequency range $f^{*} \ll 1$ with the normalized shear modulus given in Eq. (15), showing a constant value in addition to a fractional half-order normalized frequency dependence due to the physical cross-links, similar to the associated Rouse mode behaviour [59]. In time domain, the relaxation function is identical to an Abel operator kernel (10) while displaying a normalized time dependence of negative fractional order $-1 / 2$, being the long time limit of a Mittag-Leffler/scaled complementary error function

- Covers the mid-frequency range $f^{*} \approx 1$ showing a maximum normalized loss modulus according to Eq. (17) due to the maximum dissipation at the adhesion-deadhesion activities of the physical cross-links as explained by e.g. Czarnecki et al. [15]. In the relaxation time domain, maximum dissipation is displayed at the normalized relaxation time equal to 1 ; physically interpreted as the optimal normalized relaxation time for the adhesion-deadhesion activities of the physical cross-links [58].

- Covers the high-frequency range $f^{*} \gg 1$ with the normalized shear modulus given in Eq. (18), showing an elastic limit where almost all physical cross-links are fully active, in addition to the chemical crosslinks [15]. In the time domain, the relaxation function shows a stretched exponential function behaviour with a very fast decay at increasing normalized time (11), in contrast to the significantly slower decay at increasing normalized time of the Abel operator kernel (10) at the long normalized time limit

Physically, the wide spectrum of the normalized time scales in the applied relaxation Mittag-Leffler/scaled complementary error function, as compared to the ordinary exponential relaxation function, allows for a wider range of normalized relaxation times most likely found in the real, doubly cross-linked, single-network hydrogels at adhesion-deadhesion activities of the physical cross-links, thus giving a richer structure to the model. Interestingly, the 3-parameter model (19) covers the full normalized frequency range and matches measurements results remarkably well while applying only three material parameters that are physically quantifiable, namely

1. The frequency for the maximum loss modulus $f_{\mathrm{a} \mid \mathrm{d}}$, tunable by selecting a proper metal ion to modify the kinetics and thermodynamics of the adhesion-deadhesion activity of the physical cross-links [15].

2. The equilibrium elastic modulus $\mu_{\infty}$, tunable by selecting a suitable chemical cross-link density [15] and, finally,

3. The relaxation intensity $\triangle$, tunable by selecting a suitable maximally active physical-to-chemical cross-link density ratio [15].

The 3-parameter model is extended into a 4-parameter model by including a fourth parameter;

4. the chemical Rouse stress intensity factor $\mathcal{C}$.

The resulting 4-parameter model (28) covers the full normalized frequency range and matches measurements results remarkably well while also displaying a Rouse-type behaviour of the normalized chemical loss modulus with a normalized frequency dependence of the fractional order $1 / 2$ throughout the normalized frequency range and a slight increase of the normalized chemical storage modulus with normalized frequency in accordance with those of measurements.

The simple, effective visco-elastic 3- and 4-parameter models are suitable in predicting the mechanical properties of tough, doubly cross-linked, single-network PVA hydrogels with application potentials in tissue and noise abatement engineering. The 4-parameter model is preferable while more accurately modelling the normalized frequency dependence of the normalized chemical loss modulus. Nonetheless, the 3-parameter model results in satisfactory results since the normalized chemical loss modulus is substantially smaller than the other normalized modula (physical and chemical) over the considered normalized frequency range while, in addition, evidently not requiring any preparations nor measurements for the determination of the material parameter $\mathcal{C}$.

Acknowledgements Open access funding provided by Royal Institute of Technology.

Open Access This article is licensed under a Creative Commons Attribution 4.0 International License, which permits use, sharing, adaptation, distribution and reproduction in any medium or format, as long as you give appropriate credit to the original author(s) and the source, provide a link to the Creative Commons licence, and indicate if changes were made. The images or other third party material in this article are included in the article's Creative Commons licence, unless indicated otherwise in a credit line to the material. If material is not included in the article's Creative Commons licence and your intended use is not permitted by statutory regulation or exceeds the permitted use, you will need to obtain permission directly from the copyright holder. To view a copy of this licence, visit http://creativecommons.org/licenses/by/4.0/. 


\section{References}

1. Ahmed, E.M.: Hydrogel: preparation, characterization, and applications: a review. J. Adv. Res. 6, 105-121 (2015). https:// doi.org/10.1016/j.jare.2013.07.006

2. Ahmed, S., Nakajima, T., Kurokawa, T., Haque, M.A., Gong, J.P.: Brittle-ductile transition of double network hydrogels: mechanical balance of two networks as the key factor. Polymers 5, 914-923 (2014). https://doi.org/10.1016/j.polymer.2013. 12.066

3. Bagley, R.L., Torvik, P.J.: Fractional calculus—a different approach to the analysis of viscoelastically damped structures. AIAA J. 21, 741-748 (1983). https://doi.org/10.2514/3.8142

4. Bagley, R.L., Torvik, P.J.: A theoretical basis for the application of fractional calculus to viscoelasticity. J. Rheol. 27, 201-210 (1983). https://doi.org/10.1122/1.549724

5. Bagley, R.L., Torvik, P.J.: On the fractional calculus model of viscoelastic behavior. J. Rheol. 30, 133-155 (1986). https:// doi.org/10.1122/1.549887

6. Bai, R., Yang, J., Morelle, X.P., Yang, C., Suo, Z.: Fatigue fracture of self-recovery hydrogels. ACS Macro Lett. 7, 312-317 (2018). https://doi.org/10.1021/acsmacrolett.8b00045

7. Bai, R., Yang, Q., Tang, J., Morelle, X.P., Vlassak, J., Suo, Z.: Fatigue fracture of tough hydrogels. Extreme Mech. Lett. 15, 91-96 (2017). https://doi.org/10.1016/j.eml.2017.07.002

8. Bakarich, S.E., Pidcock, G.C., Balding, P., Stevens, L., Calvert, P.: Recovery from applied strain in interpenetrating polymer network hydrogels with ionic and covalent cross-links. Soft Matter 8, 9985-9988 (2012). https://doi.org/10.1039/ c2sm26745d

9. Birgersson, E., Li, H., Wu, S.: Transient analysis of temperature-sensitive neutral hydrogels. J. Mech. Phys. Solids 56, 444-466 (2008). https://doi.org/10.1016/j.jmps.2007.05.014

10. Blom, P., Kari, L.: A nonlinear constitutive audio frequency magneto-sensitive rubber model including amplitude, frequency and magnetic field dependence. J. Sound Vib. 330, 947-954 (2011). https://doi.org/10.1016/j.jsv.2010.09.010

11. Branca, C., Crupi, C., D’Angelo, G., Khouzami, K., Rifici, S., Visco, A., Wanderlingh, U.: Effect of montmorillonite on the rheological properties of dually crosslinked guar gum-based hydrogels. J. Appl. Polym. Sci. 132, 41373 (2015). https://doi. org/10.1002/app.41373

12. Buwalda, S.J., Boere, K.W.M., Dijkstra, P.J., Feijen, J., Vermonden, T., Hennink, W.E.: Hydrogels in a historical perspective: from simple networks to smart materials. J. Controlled Release 190, 254-273 (2014). https://doi.org/10.1016/j.jconrel.2014. 03.052

13. Carlsson, L., Rose, S., Hourdet, D., Marcellan, A.: Nano-hybrid self-crosslinked PDMA/silica hydrogels. Soft Matter 6, 3619-3631 (2010). https://doi.org/10.1039/c0sm00009d

14. Creton, C.: 50th anniversary perspective: networks and gels: soft but dynamic and tough. Macromolecules 50, 8297-8316 (2017). https://doi.org/10.1021/acs.macromol.7b01698

15. Czarnecki, S., Rossow, T., Seiffert, S.: Hybrid polymer-network hydrogels with tunable mechanical response. Polymers 8, 82 (2016). https://doi.org/10.3390/polym8030082

16. Gedde, U.W.: Polymer Physics. Springer, Dordrecht (1999)

17. Gil-Negrete, N., Vinolas, J., Kari, L.: A nonlinear rubber material model combining fractional order viscoelasticity and amplitude dependent effects. J. Appl. Mech. 76, 011009 (2009). https://doi.org/10.1115/1.2999454

18. Gong, J.P.: Why are double network hydrogels so tough? Soft Matter 6, 2583-2590 (2010). https://doi.org/10.1039/b924290b

19. Gong, J.P., Katsuyama, Y., Kurokawa, T., Osada, Y.: Double-network hydrogels with extremely high mechanical strength. Adv. Mater. 15, 1155-1158 (2003). https://doi.org/10.1002/adma.200304907

20. Gross, B.: Mathematical Structure of the Theories of Viscoelasticity. Hermann and Cie, Paris (1953)

21. Guo, J., Long, R., Mayumi, K., Hui, C.Y.: Mechanics of a dual cross-link gel with dynamic bonds: steady state kinetics and large deformation effects. Macromolecules 49, 3497-3507 (2016). https://doi.org/10.1021/acs.macromol.6b00421

22. Hao, J., Weiss, R.A.: Mechanical behavior of hybrid hydrogels composed of a physical and a chemical network. Polymer 54, 2174-2182 (2013). https://doi.org/10.1016/j.polymer.2013.01.052

23. Haque, M.A., Kurokawa, T., Gong, J.P.: Super tough double network hydrogels and their application as biomaterials. Polymers 53, 1805-1822 (2012). https://doi.org/10.1016/j.polymer.2012.03.013

24. Haraguchi, K., Li, H.J., Xu, Y., Li, G.: Copolymer nanocomposite hydrogels: unique tensile mechanical properties and network structures. Polymer 96, 94-103 (2016). https://doi.org/10.1016/j.polymer.2016.04.039

25. Harrass, K., Krüger, R., Möller, M., Albrecht, K., Groll, J.: Mechanically strong hydrogels with reversible behaviour under cyclic compression with MPa loading. Soft Matter 9, 2869-2877 (2013). https://doi.org/10.1039/c2sm27603h

26. Haubold, H.J., Mathai, A.M., Saxena, R.K.: Mittag-Leffler functions and their applications. J. Appl. Math. (2011). https:// doi.org/10.1155/2011/298628

27. Henderson, K.J., Zhou, T.C., Otim, K.J., Shull, K.R.: Ionically cross-linked triblock copolymer hydrogels with high strength. Macromolecules 43, 6193-6201 (2010). https://doi.org/10.1021/ma100963m

28. Hyland, L.L., Taraban, M.B., Feng, Y., Hammouda, B., Yu, Y.B.: Viscoelastic properties and nanoscale structures of composite oligopeptide-polysaccharide hydrogels. Biopolymers 97, 177-188 (2012). https://doi.org/10.1002/bip.21722

29. Kari, L.: On the waveguide modelling of dynamic stiffness of cylindrical vibration isolators. Part I: the model, solution and experimental comparison. J. Sound Vib. 244, 211-233 (2001). https://doi.org/10.1006/jsvi.2000.3468

30. Kari, L.: On the dynamic stiffness of preloaded vibration isolators in the audible frequency range: modeling and experiments. J. Acoust. Soc. Am. 113, 1909-1921 (2003). https://doi.org/10.1121/1.1557214

31. Kari, L.: Dynamic stiffness of chemically and physically ageing rubber vibration isolators in the audible frequency range. Part 1: constitutive equations. Contin. Mech. Thermodyn. 29, 1027-1046 (2017). https://doi.org/10.1007/s00161-017-05697

32. Kari, L.: Dynamic stiffness of chemically and physically ageing rubber vibration isolators in the audible frequency range. Part 2: waveguide solution. Contin. Mech. Thermodyn. 29, 1047-1059 (2017). https://doi.org/10.1007/s00161-017-0573-y 
33. Kari, L., Eriksson, P., Stenberg, B.: Dynamic stiffness of natural rubber cylinders in the audible frequency range using wave guides. Kaut. Gummi Kunstst. 54, 106-111 (2001)

34. Karobi, S.N., Sun, T.L., Kurokawa, T., Luo, F., Nakajima, T., Nonoyama, T., Gong, J.P.: Creep behavior and delayed fracture of tough polyampholyte hydrogels by tensile test. Macromolecules 49, 5630-5636 (2016). https://doi.org/10.1021/ acs.macromol.6b01016

35. Keshavarz, B., Divoux, T., Manneville, S., McKinley, G.H.: Nonlinear viscoelasticity and generalized failure criterion for polymer gels. ACS Macro Lett. 6, 663-667 (2017). https://doi.org/10.1021/acsmacrolett.7b00213

36. Koeller, R.C.: Applications of fractional calculus to the theory of viscoelasticity. J. Appl. Mech. 51, 299-307 (1984). https:// doi.org/10.1115/1.3167616

37. Kurnia, J.C., Birgersson, E., Mujumdar, A.S.: Finite deformation of fast-response thermo-sensitive hydrogels-a computational study. Polymer 53, 2500-2508 (2012). https://doi.org/10.1016/j.polymer.2012.03.049

38. Li, J., Illeperuma, W.R.K., Suo, Z., Vlassak, J.J.: Hybrid hydrogels with extremely high stiffness and toughness. ACS Macro Lett. 3, 520-523 (2014). https://doi.org/10.1021/mz5002355

39. Lin, P., Ma, S., Wang, X., Zhou, F.: Molecularly engineered dual-crosslinked hydrogel with ultrahigh mechanical strength, toughness, and good self-recovery. Adv. Mater. 27, 2054-2059 (2015). https://doi.org/10.1002/adma.201405022

40. Lin, W.C., Fan, W., Marcellan, A., Hourdet, D., Creton, C.: Large strain and fracture properties of poly(dimethylacrylamide)/silica hybrid hydrogels. Macromolecules 43, 2554-2563 (2010). https://doi.org/10.1021/ ma901937r

41. Liu, M., Guo, J., Hui, C.Y., Creton, C., Narita, T., Zehnder, A.: Time-temperature equivalence in a PVA dual cross-link self-healing hydrogel. J. Rheol. 62, 991-1000 (2018). https://doi.org/10.1122/1.5029466

42. Long, R., Mayumi, K., Creton, C., Narita, T., Hui, C.Y.: Time dependent behavior of a dual cross-link self-healing gel: theory and experiments. Macromolecules 47, 7243-7250 (2014). https://doi.org/10.1021/ma501290h

43. Long, R., Mayumi, K., Creton, C., Narita, T., Hui, C.Y.: Rheology of a dual crosslink self-healing gel: theory and measurement using parallel-plate torsional rheometry. J. Rheol. 59, 643-665 (2015). https://doi.org/10.1122/1.4915275

44. Lu, T., Wang, J., Yang, R., Wang, T.J.: A constitutive model for soft materials incorporating viscoelasticity and mullins effect. J. Appl. Mech. (2017). https://doi.org/10.1115/1.4035180

45. Luo, F., Sun, T.L., Nakajima, T., Kurokawa, T., Zhao, Y., Ihsan, A.B., Guo, H.L., Li, X.F., Gong, J.P.: Crack blunting and advancing behaviors of tough and self-healing polyampholyte hydrogel. Macromolecules 47, 6037-6046 (2014). https://doi. org/10.1021/ma5009447

46. Machado, J.T., Kiryakova, V., Mainardi, F.: Recent history of fractional calculus. Commun. Nonlinear Sci. Numer. Simul. 16, 1140-1153 (2011). https://doi.org/10.1016/j.cnsns.2010.05.027

47. Mao, Y., Lin, S., Zhao, X., Anand, L.: A large deformation viscoelastic model for double-network hydrogels. J. Mech. Phys. Solids 100, 103-130 (2017). https://doi.org/10.1016/j.jmps.2016.12.011

48. Mayumi, K., Guo, J., Narita, T., Hui, C.Y., Creton, C.: Fracture of dual crosslink gels with permanent and transient crosslinks. Extreme Mech. Lett. 6, 52-59 (2016). https://doi.org/10.1016/j.eml.2015.12.002

49. Mayumi, K., Marcellan, A., Ducouret, G., Creton, C., Narita, T.: Stress-strain relationship of highly stretchable dual cross-link gels: separability of strain and time effect. ACS Macro Lett. 2, 1065-1068 (2013). https://doi.org/10.1021/mz4005106

50. Narita, T., Mayumi, K., Ducouret, G., Hébraud, P.: Viscoelastic properties of poly(vinyl alcohol) hydrogels having permanent and transient cross-links studied by microrheology, classical rheometry, and dynamic light scattering. Macromolecules 46, 4174-4183 (2013). https://doi.org/10.1021/ma400600f

51. Nicol, E., Nicolai, T., Zhao, J., Narita, T.: Photo-cross-linked self-assembled poly(ethylene oxide)-based hydrogels containing hybrid junctions with dynamic and permanent cross-links. ACS Macro Lett. 7, 683-687 (2018). https://doi.org/10.1021/ acsmacrolett.8b00317

52. Ou, K., Dong, X., Qin, C., Ji, X., He, J.: Properties and toughening mechanisms of PVA/PAM double-network hydrogels prepared by freeze-thawing and anneal-swelling. Mater. Sci. Eng. C 77, 1017-1026 (2017). https://doi.org/10.1016/j.msec. 2017.03.287

53. Peak, C.W., Wilker, J.J., Schmidt, G.: A review on tough and sticky hydrogels. Colloids Polym. Sci. 291, 2031-2047 (2013). https://doi.org/10.1007/s00396-013-3021-y

54. Pritz, T.: Analysis of four-parameter fractional derivative model of real solid materials. J. Sound Vib. 195, 103-115 (1996). https://doi.org/10.1006/jsvi.1996.0406

55. Pritz, T.: Verification of local kramers-kronig relations for complex modulus by means of fractional derivative model. J. Sound Vib. 228, 1145-1165 (1999). https://doi.org/10.1006/jsvi.1999.2495

56. Pritz, T.: Five-parameter fractional derivative model for polymeric damping materials. J. Sound Vib. 265, 935-952 (2003). https://doi.org/10.1016/S0022-460X(02)01530-4

57. Ren, C., MacKenzie, A.R.: Closed-form approximations to the error and complementary error functions and their applications in atmospheric science. Atmos. Sci. Lett. 8, 70-73 (2007). https://doi.org/10.1002/asl.154

58. Rose, S., Dizeux, A., Narita, T., Hourdet, D., Marcellan, A.: Time dependence of dissipative and recovery processes in nanohybrid hydrogels. Macromolecules 46, 4095-4104 (2013). https://doi.org/10.1021/ma400447j

59. Rouse Jr., P.E.: A theory of the linear viscoelastic properties of dilute solutions of coiling polymers. J. Chem. Phys. 2, 1272-1280 (1953). https://doi.org/10.1063/1.1699180

60. Shao, C., Chang, H., Wang, M., Xu, F., Yang, J.: High-strength, tough, and self-healing nanocomposite physical hydrogels based on the synergistic effects of dynamic hydrogen bond and dual coordination bonds. ACS Appl. Mater. Interfaces $\mathbf{9}$, 28305-28318 (2017). https://doi.org/10.1021/acsami.7b09614

61. Sjöberg, M., Kari, L.: Non-linear behavior of a rubber isolator system using fractional derivatives. Vehicle Syst. Dyn. 37, 217-236 (2002). https://doi.org/10.1076/vesd.37.3.217.3532

62. Sjöberg, M., Kari, L.: Nonlinear isolator dynamics at finite deformations: An effective hyperelastic, fractional derivative, generalized friction model. Nonlinear Dyn. 33,323-336 (2003). https://doi.org/10.1023/A:1026037703124

63. Sun, J.Y., Zhao, X., Illeperuma, W.R.K., Chaudhuri, O., Oh, K.H., Mooney, D.J., Vlassak, J.J., Suo, Z.: Highly stretchable and tough hydrogels. Nature 489, 133-136 (2012). https://doi.org/10.1038/nature11409 
64. Sun, T.L., Kurokawa, T., Kuroda, S., Ihsan, A.B., Akasaki, T., Sato, K., Haque, M.A., Nakajima, T., Gong, J.P.: Physical hydrogels composed of polyampholytes demonstrate high toughness and viscoelasticity. Nat. Mater. 12, $932-937$ (2013). https://doi.org/10.1038/NMAT3713

65. Sun, T.L., Luo, F., Kurokawa, T., Karobi, S.N., Nakajima, T., Gong, J.P.: Molecular structure of self-healing polyampholyte hydrogels analyzed from tensile behaviors. Soft Matter 11, 9355-9366 (2015). https://doi.org/10.1039/c5sm01423a

66. Torvik, P.J., Bagley, R.L.: On the appearance of the fractional derivative in the behavior of real materials. J. Appl. Mech. 51, 294-298 (1984). https://doi.org/10.1115/1.3167615

67. Wang, B., Kari, L.: Modeling and vibration control of a smart vibration isolation system based on magneto-sensitive rubber. Smart Mater. Struct. 28, 065026 (2019). https://doi.org/10.1088/1361-665X/ab1ab4

68. Wang, B., Kari, L.: A nonlinear constitutive model by spring, fractional derivative and modified bounding surface model to represent the amplitude, frequency and the magnetic dependency for magneto-sensitive rubber. J. Sound Vib. 438, 344-352 (2019). https://doi.org/10.1016/j.jsv.2018.09.028

69. Wang, W., Zhang, Y., Liu, W.: Bioinspired fabrication of high strength hydrogels from non-covalent interactions. Prog. Polym. Sci. 71, 1-25 (2017). https://doi.org/10.1016/j.progpolymsci.2017.04.001

70. Wang, X., Hong, W.: A visco-poroelastic theory for polymeric gels. Proc. R. Soc. Lond. Ser. A 468, 3824-3841 (2012). https://doi.org/10.1098/rspa.2012.0385

71. Wharmby, A.W., Bagley, R.L.: Generalization of a theoretical basis for the application of fractional calculus to viscoelasticity. J. Rheol. 57, 1429-1440 (2013). https://doi.org/10.1122/1.4819083

72. Yang, C.H., Wang, M.X., Haider, H., Yang, J.H., Sun, J.Y., Chen, Y.M., Zhou, J., Suo, Z.: Strengthening alginate/polyacrylamide hydrogels using various multivalent cations. ACS Appl. Mater. Interfaces 5, 10418-10422 (2013). https://doi.org/10.1021/am403966x

73. Yang, J., Ma, M., Zhang, X., Xu, F.: Elucidating dynamics of precoordinated ionic bridges as sacrificial bonds in interpenetrating network hydrogels. Macromolecules 49, 4340-4348 (2016). https://doi.org/10.1021/acs.macromol.6b00874

74. Zhang, H., Peng, H., Li, Y., Xu, Y., Weng, W.: Compositional- and time-dependent dissipation, recovery and fracture toughness in hydrophobically reinforced hybrid hydrogels. Polymer 80, 130-137 (2015). https://doi.org/10.1016/j.polymer.2015.10. 052

75. Zhang, W., Hu, J., Tang, J., Wang, Z., Wang, J., Lu, T., Suo, Z.: Fracture toughness and fatigue threshold of tough hydrogels. ACS Macro Lett. 8, 17-23 (2019). https://doi.org/10.1021/acsmacrolett.8b00788

76. Zhang, W., Liu, X., Wang, J., Tang, J., Hu, J., Lu, T., Suo, Z.: Fatigue of double-network hydrogels. Eng. Fract. Mech. 187, 74-93 (2018). https://doi.org/10.1016/j.engfracmech.2017.10.018

77. Zhang, Y.S., Khademhosseini, A.: Advances in engineering hydrogels. Science 356, eaaf3627 (2017). https://doi.org/10. $1126 /$ science.aaf3627

78. Zhao, J., Mayumi, K., Creton, C., Narita, T.: Rheological properties of tough hydrogels based on an associating polymer with permanent and transient crosslinks: effects of crosslinking density. J. Rheol. 61, 1371-1383 (2017). https://doi.org/10. $1122 / 1.4997589$

79. Zhao, X.: A theory for large deformation and damage of interpenetrating polymer networks. J. Mech. Phys. Solids 60, 319-332 (2012). https://doi.org/10.1016/j.jmps.2011.10.005

80. Zhao, X.: Multi-scale multi-mechanism design of tough hydrogels: building dissipation into stretchy networks. Soft Matter 10, 672-687 (2014). https://doi.org/10.1039/c3sm52272e

81. Zhong, M., Liu, Y.T., Liu, X.Y., Shi, F.K., Zhang, L.Q., Zhu, M.F., Xie, X.M.: Dually cross-linked single network poly(acrylic acid) hydrogels with superior mechanical properties and water absorbency. Soft Matter 12, 5420-5428 (2016). https://doi. org/10.1039/c6sm00242k

82. Zhou, X., Guo, B., Zhang, L., Hu, G.H.: Progress in bio-inspired sacrificial bonds in artificial polymeric materials. Chem. Soc. Rev. 46, 6301-6329 (2017). https://doi.org/10.1039/c7cs00276a

83. Zou, X., Kui, X., Zhang, R., Zhang, Y., Wang, X., Wu, Q., Chen, T., Sun, P.: Viscoelasticity and structures in chemically and physically dual-cross-linked hydrogels: insights from rheology and proton multiple-quantum NMR spectroscopy. Macromolecules 50, 9340-9352 (2017). https://doi.org/10.1021/acs.macromol.7b01854

Publisher's Note Springer Nature remains neutral with regard to jurisdictional claims in published maps and institutional affiliations. 